\title{
COMPLETE FUNCTION SPACES
}

\author{
R.A. MCCOY \\ Department of Mathematics \\ Virginia Polytechnic Institute and State University \\ Blacksburg, Virginia 24061-4097 U.S.A. \\ (Received November 17, 1982)
}

\begin{abstract}
A study is made of certain completeness properties of the space of all continuous real-valued functions on a space, where this function space has the compact-open topology.
\end{abstract}

KEY WORDS AND PHRASES. Function space, compact-open topology, Čech-complete, Baire space.

1980 MATHEMATICS SUBJECT CLASSIFICATION CODE. $54 C 35$.

\section{INTRODUCTION.}

The set of all real-valued continuous functions defined on a topological space $\mathrm{X}$, denoted by $\mathrm{C}(\mathrm{X})$, has a number of natural topologies which have been well-used. In this paper, we study the "completeness" of one of these kinds of function spaces. Whenever $\mathrm{C}(\mathrm{X})$ has the topology of uniform covergence then it is always completely metrizable, so there is no question about the "completeness" in this case. Whenever $\mathrm{C}(\mathrm{X})$ has the topology of pointwise convergence, then the "completeness" has been studied in this case in [4]. On the other hand whenever $C(X)$ has the compact-open topology, which in many circumstances is the best or most natural topology, then the situation concerning "completeness" is not very well understood except in special cases. We study the "completeness" of $\mathrm{C}(\mathrm{X})$ with the compact-open topology, primarily for first countable spaces $X$. The general situation remains unknown. Throughout this paper all spaces $X$ are completely regular Hausdorff spaces, and the function spaces, $\mathrm{C}(\mathrm{X})$, all have the compact-open topology.

Let $F(X)$ denote the set of all real-valued functions on $X$ (not necessarily continuous). If $A \subseteq X$ and $V \subseteq R$, the space of real numbers, then we use the notation $[A, V]$ to denote the set $\{f \in F(X) \mid f(A) \subseteq V\}$. Then the compact-open topology on $F(X)$ is 
the topology generated by the subbase $\{[\mathrm{A}, \mathrm{V}] \mid \mathrm{A}$ is a compact subset of $\mathrm{X}$ and $\mathrm{V}$ is an open subset of $R$ \}. Now $C(X)$ is a subspace of $F(X)$, and when we are working with $C(X)$, we use $[A, V] \cap C(X)$. Note that $F(X)$ is a Hausdorff space, and that $C(X)$ is completely regular.

The weakest kind of completeness property is that of being of second category in itself (i.e., a space which cannot be written as a countable union of nowhere dense subsets of itself). In fact by a completeness property we mean one that lies between that of being of second category in itself and that of being completely metrizable. For function spaces, $\mathrm{C}(\mathrm{X})$ is of second category in itself if and only if it is a Baire space (i.e., every nonempty open subspace is of second category in itself). This is because $\mathrm{C}(\mathrm{X})$ is homogeneous.

The strongest kind of completeness property is that of being completely metrizable. To begin with, a function space $C(X)$ is metrizable if and only if $X$ is hemicompact [1]. The space $X$ is hemicompact provided it has a sequence of compact subsets such that every compact subset of $X$ is contained in some member of this sequence Every first countable hemicompact space is locally compact, and every locally compact Lindelöf space is hemicompact. Now it is known that $C(X)$ is completely metrizable if and only if $\mathrm{X}$ is a hemicompact $k$-space [2].

2. MAIN RESULTS.

We begin our study with a necessary condition that $C(X)$ be a Baire space.

THEOREM 1. Let $X$ contain a point $x_{0}$ such that

(i) $x_{0}$ has no compact neighborhood in $x$, and

(ii) $x_{0}$ is contained in a compact set $A_{0}$ which has a countable base in $x$. Then $C(X)$ is of first category in itself.

PROOF. Let $\mathrm{U}_{1} \supseteq \overline{\mathrm{U}}_{2} \supseteq \mathrm{U}_{2} \supseteq \overline{\mathrm{U}}_{3} \supseteq \mathrm{U}_{3} \supseteq \ldots$ be a countable base for $\mathrm{A}_{0}$. For each $\mathrm{n}$, let $\mathrm{A}_{\mathrm{n}}=\overline{\mathrm{U}}_{\mathrm{n}} \backslash \mathrm{U}_{\mathrm{n}+1}$. There is some integer $\mathrm{k}_{1}$ such that $\mathrm{A}_{\mathrm{k}_{1}}$ is not compact, since otherwise $A_{0} \cup A_{1} \cup A_{2} \cup \ldots$ would be a compact neighborhood of $x_{0} \cdot$ In fact we can find an increasing sequence $k_{1}, k_{2}, \ldots$ such that each $A_{k_{n}}$ is not compact. For each $n$, let $G_{n}=U\left\{[\{a\},(n, n+1)] \mid a \varepsilon A_{k_{n}}\right\}$, which is an open dense subset of $c(x)$.

Suppose there exists an $f \varepsilon G_{1} \cap G_{2} \cap \ldots$. Then for each $n$, there is an $a_{n} \varepsilon A_{k_{n}}$ such that $f\left(a_{n}\right) \varepsilon(n, n+1)$. Now $f\left(A_{0}\right)$ is contained in some bounded open interval 
$(-M, M)$. But then there is an $n>M$ such that $\bar{U}_{k_{n}} \subseteq f^{-1}((-M, M))$, so that $f\left(a_{n}\right)>n>M$, which is a contradiction. Therefore $G_{1} \cap G_{2} \cap \ldots=\emptyset$, so that $C(X)$ is of first category in itself.

The following corollary of Theorem 1 can be found in [3].

COROLLARY 1. If $X$ is first countable and $C(X)$ is a Baire space, then $X$ is locally compact.

This has a partial converse which was pointed out in [3].

THEOREM 2. If $X$ is a locally compact paracompact space, then $C(X)$ is a Baire space.

PROOF. The space $X$ is the topological sum of locally compact $\sigma$-compact spaces $\mathrm{X}_{\alpha}$, $\alpha \varepsilon A$. Each $\mathrm{C}\left(\mathrm{X}_{\alpha}\right)$ is completely metrizable, so that $\Pi\left\{\mathrm{C}\left(\mathrm{X}_{\alpha}\right) \mid \alpha \varepsilon A\right\}$ is a Baire space. But this product is homeomorphic to $\mathrm{C}(\mathrm{X})$.

There are many other well-studied completeness properties. One of these is the property of being pseudo-complete. A space X is pseudo-complete provided it has a sequence $\left\{P_{n}\right\}$ of pseudo-bases such that whenever $P_{n} \varepsilon P_{n}$ is such that each $\bar{P}_{n+1} \subseteq P_{n}$, then $\mathrm{P}_{1} \cap \mathrm{P}_{2} \cap \ldots \neq \varnothing$. This is a productive property, so that the conclusion that $\mathrm{C}(\mathrm{X})$ be a Baire space in Theorem 2 can be replaced with the stronger conclusion that $\mathrm{C}(\mathrm{X})$ is a pseudo-complete. In fact we have the following corollary of Theorems 1 and 2 .

COROLLARY 2. If $\mathrm{X}$ is a first countable paracompact space, then the following are equivalent.

1. $C(X)$ is a Baire space.

2. $\mathrm{C}(\mathrm{X})$ is a pseudo-complete.

3. $\mathrm{C}(\mathrm{X})$ is the product of completely metrizable spaces.

4. $\mathrm{X}$ is locally compact.

Another completeness property which is stronger than that of being pseudo-

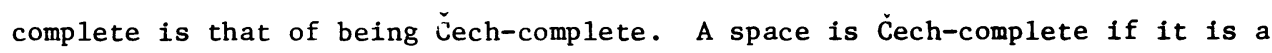
$\mathrm{G}_{\delta}$-subset of its Stone-Čech compactification. Corollary 1 has an analog for this property. We first establish an analog to Lemma 8.7 in [4].

LEMMA 1. If $C(X)$ contains a nonempty $G_{\delta}$-subset of $F(X)$, then $X$ is the topological sum of a $\sigma$-compact space and a discrete space. 
PROOF. Let $f \varepsilon C(X)$ and let $\left\{W_{n}\right\}$ be a sequence of basic open subsets of $F(X)$ such that $f \varepsilon W_{1} \cap W_{2} \cap \ldots \subseteq C(X)$. To be specific, suppose that each $W_{n}=\left[A_{n 1}, V_{n 1}\right] \cap \ldots n$ $\left[A_{n k}, V_{n k}\right]$. Define $A=u\left\{A_{n i} \mid n=1,2, \ldots\right.$, and $\left.i=1, \ldots, k_{n}\right\}$, and let $B=X \backslash A$. Note that if $g \in F(X)$ with $f_{A}=\left.f\right|_{A}$, then $g \varepsilon C(X)$.

Let $\mathrm{p}$ be a non-isolated point of $\mathrm{X}$. Suppose by way of contradiction that pæA. Then define $g: X \rightarrow R$ by $g(x)=f(x)$ for $x \in X \backslash\{p\}$ and $g(p)=f(p)+1$. Since $d A=f \mid A$, then $g \varepsilon C(X)$. But since $X \backslash\{p\}$ is dense in Hausdorff space $X$, then $f=g$, which is a contradiction. Therefore, A contains all the non-isolated points of $X$. In particu$\operatorname{lar} A$ is closed in $X$ and $B$ is discrete.

Again, by way of contradiction suppose that B has some accumulation point p, which would necessarily be in $A$. Define $g: X \rightarrow R$ by $g(x)=f(x)$ if $x \in A$ and $g(x)=f(p)$ +1 if $x \in B$. Again since $d_{A}=\left.f\right|_{A}$ then $g \varepsilon C(X)$. Since $p$ is an accumulation point $B$, and $g$ is constant $f(p)+1$ on $B$, then $g(p)=f(p)+1$. But since $p \varepsilon A$, then $g(p)=$ $=f(p)$, which is a contradiction. Therefore B has no accumulation point, so that A is open in $\mathrm{X}$. Then $\mathrm{X}$ is the topological sum of the $\sigma$-compact space $\mathrm{A}$ and the discrete space $B$.

THEOREM 3. If $X$ is first countable and $C(X)$ is Cech-complete, then $X$ is hemicompact.

PROOF. Since $\mathrm{C}(\mathrm{X})$ is Lech-complete and dense in Hausdorff space $\mathrm{F}(\mathrm{X})$, then $\mathrm{C}(\mathrm{X})$ is a $G_{\delta}$-subset of $F(X)$. By Lemma $1, X$ is the topological sum of $\sigma$-compact space $A$ and discrete space $B$. Then $C(X)$ is homeomorphic to $C(A) \times C(B)=C(A) \times R^{B}$. Now each of $C(A)$ and $R^{B}$ must be Cech-complete. In the first place this implies that $B$ is countable. Also since A is first countable, then it is locally compact by Corollary 1. Finally a locally compact $\sigma$-compact space is hemicompact.

COROLLARY 3. If $\mathrm{X}$ is first countable then the following are equivalent.

1. $C(X)$ is Čech-complete.

2. $\mathrm{C}(\mathrm{X})$ is completely metrizable.

3. $\mathrm{X}$ is hemicompact.

The Cech-complete property implies that of being a k-space (compactly generated space). Although the $\mathrm{k}$-space property is not really a completeness property since 
such spaces need not be Baire spaces, still for function spaces with the compact-open topology, there is a relationship between these properties (which is not completely known). We see this with our last theorem. Some of these ideas can be found in [5]. LEMMA 2. If $X$ is first countable at some non-isolated point, then $C(X)$ is not countably compact.

PROOF. Let $\left\{x_{n}\right\}$ be a sequence of distinct points of $x$ converging to some point $x_{0}$. For each $n$ define function $f_{n} \varepsilon C(X)$ satisfying:

(i) $f_{n}\left(x_{i}\right)=0$ for $i>n$,

(ii) $\mathrm{f}_{\mathrm{n}}\left(\mathrm{x}_{\mathrm{i}}\right)=1$ for $\mathrm{i} \leq \mathrm{n}$,

(iii) $\mathrm{f}_{\mathrm{n}}\left(\mathrm{x}_{0}\right)=0$.

Then the set $\left\{\mathrm{f}_{\mathrm{n}}\right\}$ is closed and discrete in $\mathrm{C}(\mathrm{X})$.

In the next lemma, $\mathrm{N}$ denotes the set of natural numbers.

LEMMA 3. If $\mathrm{X}$ is a first countable paracompact space which is not Lindelöf, then $C(X)$ contains a closed copy of $\mathrm{N}^{\mathrm{t}} \mathrm{i}$.

PROOF. Let $Y$ be the subspace of non-isolated points of $X$. First suppose that $\mathrm{Y}$ is Lindelöf. Then $\mathrm{X}$ contains an uncountable closed subspace $\mathrm{D}$ consisting of isolated points of $X$. If $Z=X \backslash D$, then $C(X)$ is homeomorphic to $C(Z) \times C(D)$. But C(D) clearly contains a closed copy of $\mathrm{N}^{N_{1}}$.

Now suppose that $\mathrm{Y}$ is not Lindelöf. Then there exists a discrete family $A$ of closed subsets of $X$ having cardinality $N_{1}$, such that int $A \cap Y \neq \varnothing$ for each $A \varepsilon A$. Define $F=\{f \varepsilon C(X) \mid f$ is supported on $\cup A\}$, and for each $A \varepsilon A$, let $F_{A}=\{f \varepsilon C(X) \mid f$ is supported on $A\}$. We see that $F$ is a closed subspace of $C(X)$ which is homeomorphic to $\Pi\left\{F_{A} \mid A \varepsilon A\right\}$. By Lemma 2, each $F_{A}$ is not countable compact, and therefore contains a closed copy of $N$. Then $F$, and also $C(X)$, must contain a closed copy of $N^{N 1}$.

THEOREM 4. If $X$ is a first countable paracompact space and $C(X)$ is a $k$-space, then $\mathrm{X}$ is hemicompact.

PROOF. First since $\mathrm{N}^{2} \mathrm{l}$ is not a $\mathrm{k}$-space, then $\mathrm{X}$ would have to be Lindelöf by Lemma 3. Since a locally compact Lindelöf space is hemicompact, it will suffice to show that $\mathrm{X}$ is locally compact.

Suppose that $\mathrm{X}$ is not locally compact at $\mathrm{x}_{0}$, and let $\mathrm{U}_{1} \supseteq \overline{\mathrm{U}}_{2} \supseteq \mathrm{U}_{2} \supseteq \ldots$ be a 
countable base at $x_{0}$. For each $n$, let $A_{n}=\bar{U}_{n} \backslash U_{n+1}$. As in the proof of Theorem 1 , there is a sequence $\left\{k_{n}\right\}$ such that each $A_{k_{n}}$ is not compact and $k_{n+1}>k_{n}+1$ for each $n$. Then for each $n$, there exists a countable closed discrete subset $\left\{a_{n}^{i} \mid i \varepsilon N\right\}$ $\subseteq A_{k_{n}}$, and there exists a discrete family of open subsets $\left\{v_{n}^{i} \mid i \varepsilon N\right\}$ such that $a_{n}^{i} \varepsilon v_{n}^{i}$ and $v_{n} \cap \vec{U}_{k_{n}+1}=\varnothing$. For each $n \geq 1$ and $i>n$, define $f_{n}^{i} \varepsilon C(X)$ satisfying:

$$
\begin{aligned}
& \text { (i) } f_{n}^{i}\left(a_{n}^{i}\right)=1, \\
& \text { (ii) } f_{n}^{i}\left(a_{i}^{i}\right)=0, \\
& \text { (iii) } f_{n}^{i}\left(x_{0}\right)=1 / n, \\
& \text { (iv) } f_{n}^{i}(x) \leq 1 / n \text { for every } x \notin v_{n}^{i} .
\end{aligned}
$$

Now define $F=\left\{f_{n}^{i} \mid 1 \leq n<i\right\}$. By Ascoli's Theorem, $K \cap F$ is finite for each compact $K \subseteq C(X)$. Also the constant 0 function is in $\bar{F} \backslash F$, so that $C(X)$ would not be a k-space.

COROLLARY 4. If $\mathrm{X}$ is a first countable paracompact space, then the following are equivalent.

1. $\mathrm{C}(\mathrm{X})$ is a k-space.

2. $C(X)$ is Čech-complete.

3. $\mathrm{C}(\mathrm{X})$ is completely metrizable.

4. $\mathrm{X}$ is hemicompact.

We give some examples showing that none of the hypotheses in the theorems on Baire spaces can be omitted.

EXAMPLE 1. Let $X$ be an uncountable sDace with exactly one non-isolated point, which has as neighborhoods those sets containing it having countable complements. Now $X$ is a Lindelöf space which is not first countable or locally compact. Also the compact subsets of $X$ are finite, so that $C(X)$ in fact has the topology of pointwise convergence. From [4], we see that $C(X)$ is pseudo-complete but not Čechcomplete. In particular we see that Corollary 1 becomes false if "first countable" is replaced by "Lindelöf."

EXAMPLE 2. Let $\mathrm{X}$ be the space of countable ordinals with the order topology. Then $\mathrm{X}$ is a first countable, locally compact space which is not paracompact. For 
each $n$, let $D_{n}$ be the set of isolated points of the $n$th derived set of $x$. Then define $G_{n}=U\left\{[\{x\},(n-1, n)] \mid x \in D_{n}\right\}$. Each $G_{n}$ is open and dense in $C(X)$. But since every function in $C(X)$ is bounded, then $G_{1} \cap G_{2} \cap \ldots=\emptyset$. Therefore, $C(X)$ is of first category in itself. Then in Theorem 2, "paracompact" cannot be omitted, or even replaced by "first countable."

Finally, there are some questions which come to mind here.

Questions :

1. Can "first countable" be omitted from Theorem 3?

2. Can "paracompact" be omitted from Theorem 4?

3. Is there a characterization of $C(X)$ being pseudo-complete which is analogous to Theorem 8.4 of [4]?

In fact the only completeness property on $\mathrm{C}(\mathrm{X})$ which has a known characterization in terms of a property on X (for general Tychonoff spaces X) is that of complete metrizability. So there are many other questions which arise naturally in this topic.

\section{REFERENCES}

1. ARENS, R.F. A Topology for Spaces of Transformations, Annals of Math. 47 (1946), 480-495.

2. BECKENSTEIN, E., NARICI, L., AND SUFFEL, C. Topological Algebras, Notas de Mathematica (60), North-Holland Publishing Co., New York, 1977.

3. LEHNER, W. Über die Bedeutung gewisser Varianten des Baire'schen Kategorienbegriffs für die Funktionenräume $C_{c}(T)$, dissertation, Ludwig-Maximilians-

4. LUTZER, D.J. AND MCCOY, R.A. Category in Function Spaces I, Pacific J. Math. $90(1980), 145-168$.

5. POL, R. Normality in Function Spaces, Fund. Math. 84 (1974), 145-155. 


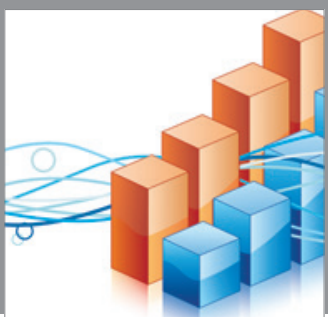

Advances in

Operations Research

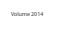

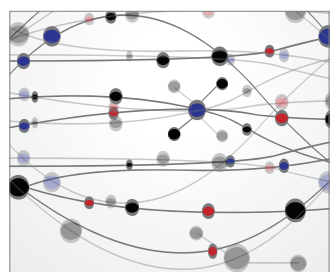

\section{The Scientific} World Journal


International Journal of

Mathematics and

Mathematical

Sciences
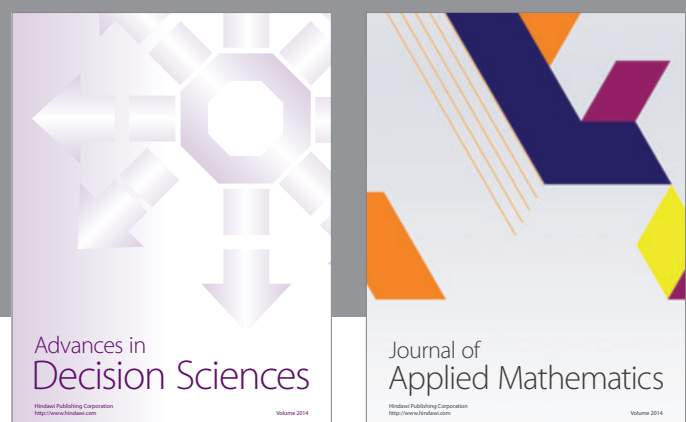

Journal of

Applied Mathematics
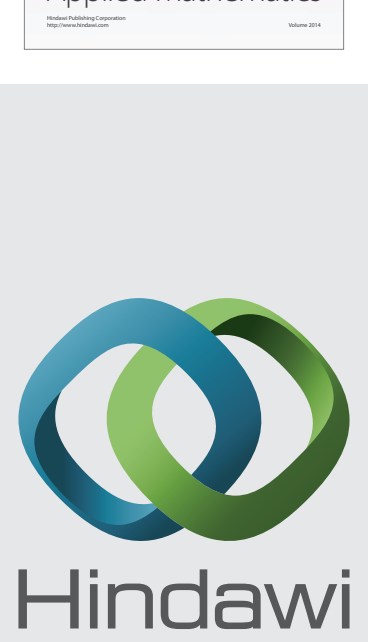

Submit your manuscripts at http://www.hindawi.com
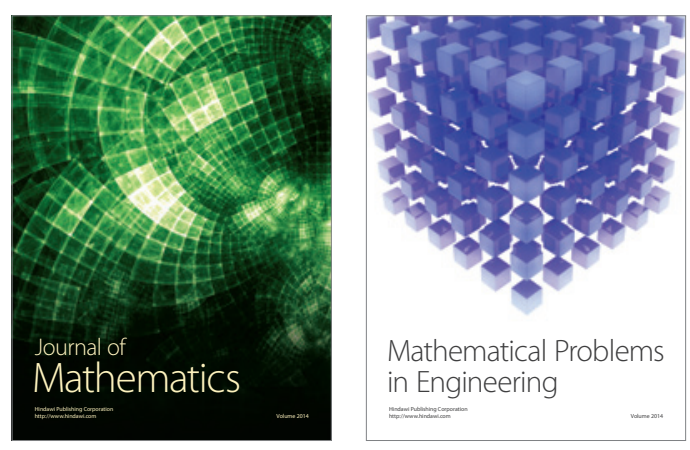

Mathematical Problems in Engineering
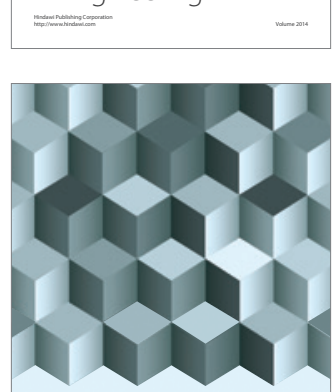

Journal of

Function Spaces
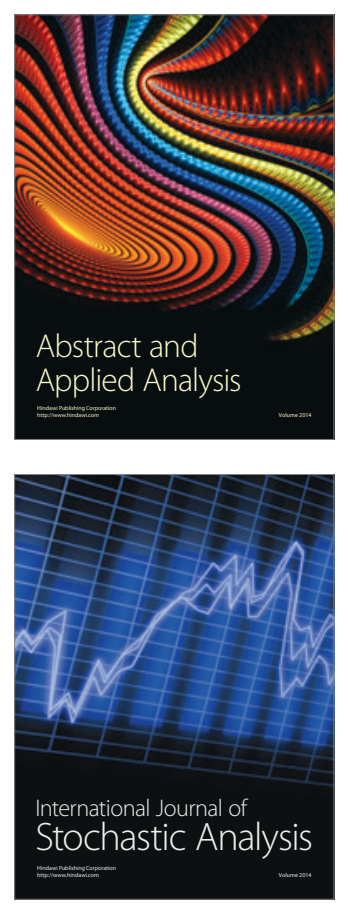

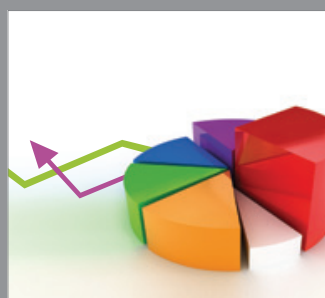

ournal of

Probability and Statistics

Promensencen
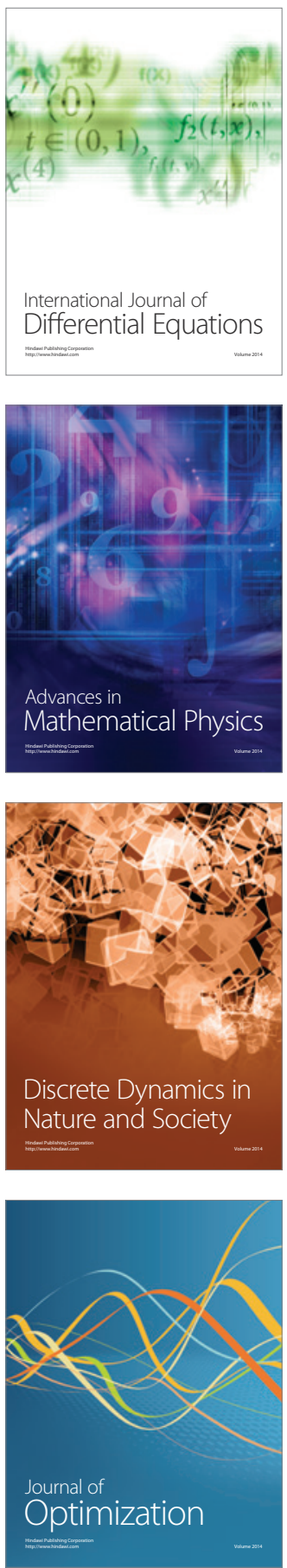